\title{
Modeling science: studying the structure and dynamics of science
}

\author{
Katy Börner • Wolfgang Glänzel • Andrea Scharnhorst • \\ Peter van den Besselaar
}

Received: 1 June 2011 / Published online: 28 June 2011

(C) Akadémiai Kiadó, Budapest, Hungary 2011

Mathematical models of the science and technology (S\&T) system have a long tradition in scientometrics. They entail models of statistical properties such as the cumulative advantage model for citation patterns by Derek de Solla Price (1976) or models of scientific processes such as the epidemics of scientific ideas by William Goffman (1966). Frequently, new modeling attempts "echo" major breakthroughs in mathematical modeling. For example, models developed in physics, economics, or the social sciences are frequently applied to the science system itself, validated using S\&T data, and interpreted by the authors of these models and their collaborators. This special issue aims to establish models of the science system as a promising area of research in scientometrics enabled by high-quality and high-coverage data, advanced data mining and modeling approaches, and new means to visualize the structure and dynamics of science at multiple levels. Models of science aim to answer questions regarding the basic mechanisms behind emergent structures such as scientific disciplines, scientific paradigms and cross-disciplinary research fronts, or the career trajectories of researchers.

The issue comprises six selected contributions, resulting from presentations at the workshop "Modeling Science-Understanding, Forecasting and Communicating The Science System," held in Amsterdam October 6-9, 2009.

- Peter Mutschke, Philipp Mayr, Philipp Schaer, and York Sure Science Models as Value-Added Services for Scholarly Information Systems

- Serge Galam Tailor Based Allocations for Multiple Authorship: A Fractional gh-Index

K. Börner

Indiana University, Bloomington, IN, USA

W. Glänzel ( $\bowtie)$

Katholieke Universiteit Leuven, Leuven, Belgium

e-mail: Wolfgang.Glanzel@econ.kuleuven.ac.be

\author{
A. Scharnhorst \\ and e-Humanities Group), Eindhoven, The Netherlands \\ P. van den Besselaar \\ Vrije Universiteit Amsterdam, Amsterdam, The Netherlands
}

The Royal Netherlands Academy of Arts and Sciences (Data Archives and Networked Services 
- Timothy S. Evans, Renaud Lambiotte, and Pietro Panzarasa Community Structure and Patterns of Scientific Collaboration in Business and Management

- M. Laura Frigotto and Massimo Riccaboni A Few Special Cases: Scientific Creativity and Network Dynamics in the Field of Rare Diseases

- Hanning Guo, Scott Weingart, and Katy Börner: Mixed-Indicators Model for Identifying Emerging Research Areas

- Christopher Watts and Nigel Gilbert: Does Cumulative Advantage Affect Collective Learning in Science? An Agent-Based Simulation

The papers in this special issue span a wide range: the possible use of information retrieval as a test-bed for models (Mutschke, Mayr, Schaer, Sure), the study of properties of new indicators such as the h-index (Galam), or measuring and modeling scientific collaboration (Evans, Lambiotte, Panzarasa), scientific creativity (Frigotto, Riccaboni), newly emerging research areas (Guo, Weingart, Börner), and learning (Watts, Gilbert). Different datasets at different scales are used to design and validate the models. In-depth, field-specific analyses as well as generic statements about the nature of scientific activity are made. Globalization and increasing specialization accompanied by interdisciplinary research, and changing institutional conditions (such as funding and tenure schemes) impact the structure and dynamics of science and models of the science system. Triangulation of methods (narratives, survey and bibliometrics indicators) is one possible answer to tackle complexity (Frigotto, Riccaboni); combining different indicators and visual analytics is another one (Guo, Weingart, Börner). Simulation models allow testing different scenarios in the space of theoretical assumptions as well as in the empirical space (Watts, Gilbert).

Many challenges remain: The majority of existing models remain unconnected. There are very few attempts to compare, synthesize, or interconnect existing models (Tabah 1999; Morris and Van der Veer Martens 2008; Scharnhorst et al. 2011). Future work should aim to integrate modeling approaches and results from different disciplines to arrive at a more comprehensive understanding of the science system.

Acknowledgments We would like to thank the authors for their professional contributions to multiple rounds of discussions and revisions, the anonymous reviewers for their expert comments, and the journal Scientometrics for editorial advice and support. This work was supported by the COST (European Cooperation in Science and Technology) action MP0801 - "Physics of Competition and Conflicts," the Royal Netherlands Academy of Arts and Sciences (KNAW), in particular the Virtual Knowledge Studio for the Humanities and Social Sciences, the James S. McDonnell Foundation, the Cyberinfrastructure for Network Science Center (http://cns.iu.edu) at Indiana University, USA, and the Rathenau Institute, The Netherlands.

\section{References}

Goffman, W. (1966). Mathematical approach to the spread of scientific ideas-the history of mast cell research. Nature, 212(5061), 449.

Morris, S. A., \& Van der Veer Martens, B. (2008). Mapping research specialties. Annual Review of Information Science and Technology, 42(1), 213-295.

Price, D. J. de Solla (1976). A general theory of bibliometric and other cumulative advantage processes. Journal of the American Society for Information Science, 27(5), 292-306.

Scharnhorst, A., Börner, K., \& Van den Besselaar, P. (Eds.) (2011). Models of Science Dynamics. Berlin: Springer.

Tabah, A. N. (1999). Literature dynamics: Studies on growth, diffusion, and epidemics. Annual Review of Information Science and Technology, 34, 249-286. 